\title{
Influence of long-term hypodynamy on spongy bone tissue in Japanese quails
}

\author{
Lucia Tarabová, Viera Almášiová, Katarína Holovská, Viera Cigánková \\ University of Veterinary Medicine and Pharmacy, Department of Anatomy, \\ Histology and Physiology, Košice, Slovak Republic
}

Received October 4, 2012

Accepted March 19, 2013

\begin{abstract}
Weightlessness can cause various damages especially on the musculoskeletal system both in animals and humans. The aim of our study was to observe the influence of simulated, long-term microgravity on the spongy bone tissue of the femur in Japanese quails. A total of 80 cockerels at the age of 2 days were exposed to simulated microgravity - hypodynamy. After days 56, 63, 90 and 180, six birds from the experimental group and six birds from the control group were euthanised. Samples for histological examination were collected from femur epiphysis. The whole femur of the other limb was used for the analysis of the calcium content. Microscopic examination showed differences between experimental and control animals in the spongy bone tissue after every day of the experiment. In the experimental animals, there were numerous, big, multinucleated cells osteoclasts, lying on the bone trabeculae surface, which were damaged. The highest difference in the calcium content in femurs between the control and experimental animals was found after 90 days of hypodynamy. This study builds on short-term hypodynamy experiments; such long periods had never been studied before in birds. Because our findings are similar to those found in osteoporotic bone tissue, it could by useful in the development of countermeasures against the negative influence of microgravity and immobilization.
\end{abstract}

Microgravity, morphology, osteoporosis, osteoclasts

Osteoporosis is a systemic skeletal disease characterized by low bone mass and microarchitectural deterioration of bone tissue, with a consequent increase in bone fragility and susceptibility to fracture (WHO 1994). Among the well known pathological conditions that can lead to development of osteoporosis are the absence of motion and weightlessness. That is why osteoporosis in connection with muscular atrophy occurs in immobilized patients and was also found in cosmonauts after longterm space missions.

In order to find out effective countermeasures to prevent the development of osteoporosis, it is necessary to know the cytological mechanism of how these changes develop, and to focus on the morphological changes in defected tissues. Experiments under real microgravity in space are demanding and very expensive, so several models for simulated microgravity on Earth - hypodynamy were created, using experimental animals such as birds, rats and monkeys. Japanese quails are small, unexacting birds with low body weight, fast individual development and high tolerance to difficult conditions, good reproduction and high production (Baumgartner and Hetenyi 2001). Thanks to their good adaptability to microgravity they are great experimental animals for simulated microgravity (Skrobanek and Hrancova 2003). In future they might be used in space shuttles during long-term space flights as producers of animal proteins for astronauts (Boda 1984, 1997).

In this work we focused on structural and ultrastructural changes in spongy bone tissue in Japanese quails and their development during different periods of long-term hypodynamy. Our results should enlarge knowledge about osteoporosis and should be useful in improvement of countermeasures to prevent expansion of these changes in immobilized patients or cosmonauts.

Address for correspondence:

MVDr. Lucia Tarabova

Department of Anatomy, Histology and Physiology

University of Veterinary Medicine and Pharmacy

Phone: +421915986737

Komenského 73,041 81 Košice, Slovak Republic 


\section{Materials and Methods}

Animals and experimental design

The experiments were carried out at the Institute of Animal Biochemistry and Genetics of the Slovak Academy of Sciences in Ivanka pri Dunaji. Forty newly hatched cockerels were used as experimental animals and forty as the control group. Both groups were kept in a windowless room with controlled ventilation and continuous lighting. The birds were fed HYD-13 commercial starter rations and fresh water ad libitum. To simulate microgravity, the birds were kept in special individual slings suspended by a flexible metal device, so they could not touch the ground with their feet, as was done before in a previous study (Skrobanek et al. 2004). The care and use of experimental animals were in accordance with the laws and regulations of the Slovak Republic and approved by the Ethics Committee of the Institute of Animal Biochemistry and Genetics, Slovak Academy of Sciences, Ivanka pri Dunaji and the State Veterinary and Food Agency (ŚVPS SR Č.K. Ro-7879/04-220/3).

\section{Microscopic examination}

After days 56, 63, 90 and 180 of hypodynamy, six randomly selected birds from each group were euthanised. One whole pelvic limb was excised from each and fixed immediately in $4 \%$ neutral formaldehyde. These samples were examined for the presence of $\mathrm{Ca}$ in the femur using flame atomic absorption spectrophotometer. Samples of femur epiphysis of the other limb were taken for light microscopy (LM) and transmission electron microscope (TEM) examination. Samples for LM, sized $0.3 \mathrm{~cm} \times 1.5 \mathrm{~cm}$, were fixed in $4 \%$ neutral formaldehyde and decalcified in buffered ethylenediaminetetraacetic acid (EDTA) at pH 7.2. After decalcification they were embedded in paraffin and cut at microtome. Sections of samples 5-10 $\mu \mathrm{m}$ thick were stained with haematoxylin-eosin, observed and photographed under light microscope (AXIO Lab A).

Samples for TEM were fixed in 3\% glutaraldehyde, stored in $0.1 \mathrm{M}$ cacodylate buffer (pH 7.2) and decalcified in buffered EDTA at $\mathrm{pH}$ 7.2. Before embedding in synthetic resin Durcupan ACM they were postfixed in $1 \%$ osmium tetraoxide in $0.2 \mathrm{M}$ cacodyle buffer and dehydrated in acetone. Semi-thin sections $(1 \mu \mathrm{m})$ were cut by ultramicrotome Tesla BS 490, stained with toluidine blue and observed under light microscope. For TEM ultra-thin sections $(90-120 \mathrm{~nm})$ were biphasic contrasted with uranyl acetate and lead citrate, observed and photographed by the electron microscope Tesla BS 500.

\section{Results}

Histological changes in the spongy bone tissue of the femur of Japanese quail were found after each stage of hypodynamy. We subjectively noticed changes in the bone matrix, occurrence of bone trabeculae, and reduction of their thickness. On days 56 and 63 of hypodynamy there were wide bone trabeculae with uneven bone matrix colour, which we assumed to be the result of decalcification (Plates IV, V, Figs 1, 2, 3, 4). The bone trabeculae had uneven, foveolated surface and were covered by large, bone resorpting osteoclast cells with foamy cytoplasm, containing 5-8 nuclei. On day 90 of hypodynamy there were bone trabeculae strongly reduced in thickness but their surface was not so markedly foveolated (Plate VI, Fig. 5). They were covered with osteoclasts, which were in comparison with previous days smaller with fewer nuclei. After 180 days of hypodynamy there were thin bone trabeculae with only sporadic presence of osteoclasts on their surface (Plates VI, VII, Figs 6, 7). The bone marrow in each period of hypodynamy contained blood forming elements and after 180 days of experiment it contained also lipid droplets. The bone marrow of control animals contained lipid droplets in all cases. The transmission electron microscope examination revealed the following submicroscopic changes: osteoclasts had an ultrastructure typical of the intracellular matrix, which points out to higher resorption activity of these giant cells. Foamy looking cytoplasm contained many vacuoles and vesicles, confirming their massive vesicular transport activity. In the sticking place of osteoclasts with bone trabeculae, fully developed ruffled border was noticeable consisting of highly folded microvilli (Plate VII, Fig. 8).

Differences in the calcium content of the femur between control and experimental animals were most visible after 63 and 90 days of hypodynamy and should be connected with higher activity of osteoclasts and increased bone demineralization. 


\section{Discussion}

In the present study we proved that long-term hypodynamy has a negative effect on the spongy bone tissue of Japanese quails. Significant morphological findings such as increased number and size of osteoclasts, attenuation and destruction of the bone trabeculae indicated elevated resorptive activity of osteoclasts, failure in mineralization and osteosynthesis. Investigation of the calcium content in the femur supports our findings from light microscopic examination. Similar changes were found also in rats which were kept in simulated microgravity (Morey-Holton and Globus 2002; Planel 2004). Other animal experiments were performed on monkeys, which were held in a sitting position for six months. Histological examination shows reduction in the spongy bone tissue of vertebras (Planel 2004).

This study follows previous research on the Japanese quail bones conducted by Zibrin et al. (2003) with different periods of hypodynamy. Bones are not the only one tissue affected by weightlessness. Long- term experimental hypodynamy induced disorders of kidneys (Almasiova et al. 2008), muscles of the pelvic limbs (Holovska et al. 2011), adrenal glands (Cigankova et al. 2005) and morphological changes were also found in cells of the small intestine epithelium (Cigankova et al. 2007). All these studies increased our knowledge about the risks of long-term space missions, immobilization or lack of physical activity. These studies can be used for development of more effective countermeasures beside those used in osteoporosis treatment to reduce its negative influence on human and animal body.

\section{Acknowledgements}

This work was supported by the Grant Agency for Science of the Slovak Republic, VEGA 1/0334/09. We would also like to thank co-workers from SAV Ivanka pri Dunaji for their collaboration and for provision of biological materials.

\section{References}

Almasiova V, Cigankova V, Holovska K, Lenhardt L, Skrobanek P, Massanyi P, Zibrin M 2008: Effect of hypodynamy on structure and alkaline phosphatase activity of kidney in Japanese quails. Acta Vet Brno 77: 313-320

Baumgartner J, Hetenyi L 2001: Japanese quail (In Slovak). VÚŽV, Publ. house Slovenský chov, Nitra 75

Boda K 1984: Cosmic biology. Gravitation physiology (In Slovak). Publ. house SAV, Kosice 9-12

Boda K 1997: Cosmic Biology and Veterinary medicine (In Slovak). Slov Vet Cas 22: 12-16

Cigankova V, Zibrin M, Boda K, Holovska K 2005: Effect of long-term experimental hypodynamy on the adrenal glands of Japanese quails: An ultrastructural study. Bull Vet Inst Pulaway 49: 449-453

Cigankova V, Zibrin M, Lenhardt L, Almasiova V, Holovska K, Skrobanek P 2007: Effect of hypodynamy on structure and enzymatic activity of duodenal enterocytes of Japanese quail. Bull Vet Inst Pulaway 51: 161-166

Holovska K, Almasiova V, Tarabova L, Cigankova V 2011: Ultrastructural changes in the m. gastrocnemicus of Japanese quails during long-lasting hypodynamy. Folia Vet 55: 99-102

Morey-Holton ER, Globus RK 2002: Hindlimb unloading rodent model: technical aspects. J Appl Physiol 92: 1367-1377

Planel H 2004: The skeletal system and weightlessness, Animal experiments. In: Space and Life: an introduction to space biology and medicine, CRC Press, USA: 49-61

Skrobanek P, Hrancova M 2003: Adaptability of Japanese quail chicks to conditions for simulated weightlessness. Acta Vet Brno 2: 347-351

Skrobanek P, Hrbata M, Baranovska M, Jurani M 2004: Growth of Japanese quails chicks in simulated weightlessness. Acta Vet Brno 73: 157-164

WHO Study Group 1994: Assessment of fracture risk and its application to screening for postmenopausal osteoporosis. World Health Organ Tech Rep Ser 843: 1-129

Zibrin M, Boda K, Cigankova V, Kocisova J, Komorova T, Tomajkova E, Sabo V, Pivko J 2003: Long-term experimental hypodynamy affects the structure of spongy bone and osteoclasts in Japanese quail. Acta Vet Brno 72: 143 -149 
Plate IV

Tarabová L. et al.: Influence of ... pp.225-227

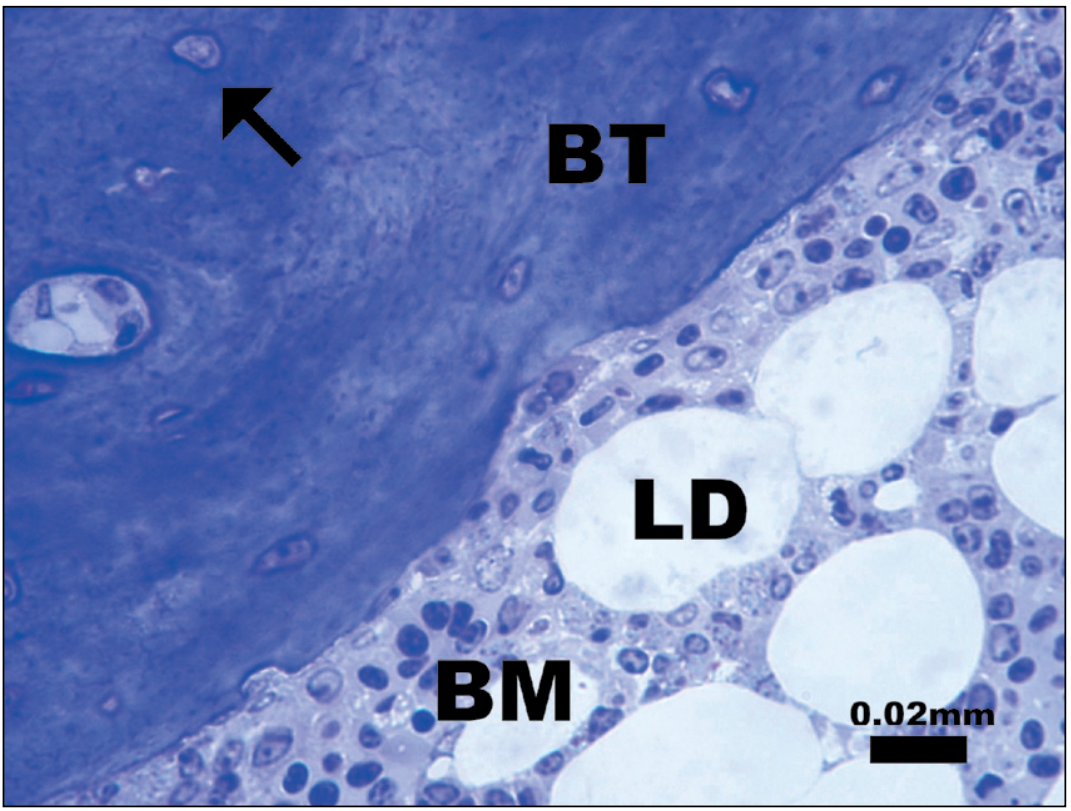

Fig. 1. Semi-thin section of the spongy bone tissue of control Japanese quail after 56 days of hypodynamy (toluidine-blue staining).

$\mathrm{BT}$ - bone trabeculae, BM - bone marrow, LD - lipid droplets, arrow - osteocyte

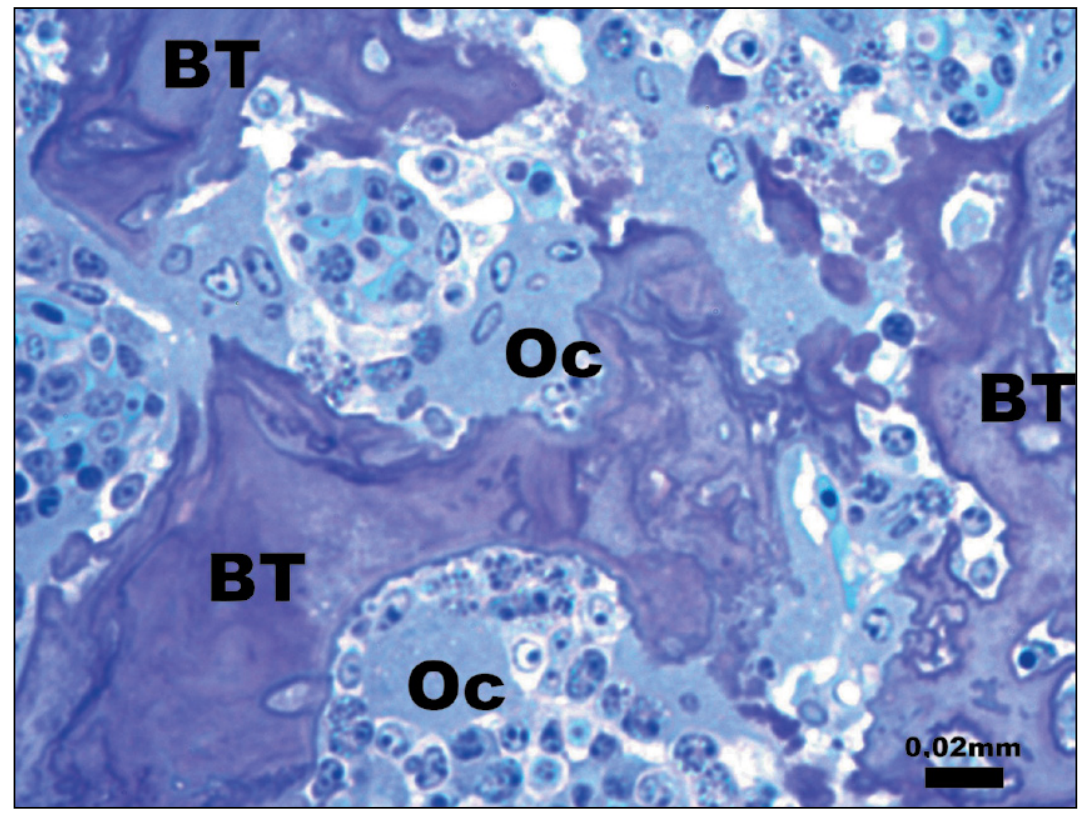

Fig. 2. Semi-thin section of the spongy bone tissue of experimental Japanese quail after 56 days of hypodynamy (toluidine-blue staining).

Oc - osteoclasts, BT - bone trabeculae 


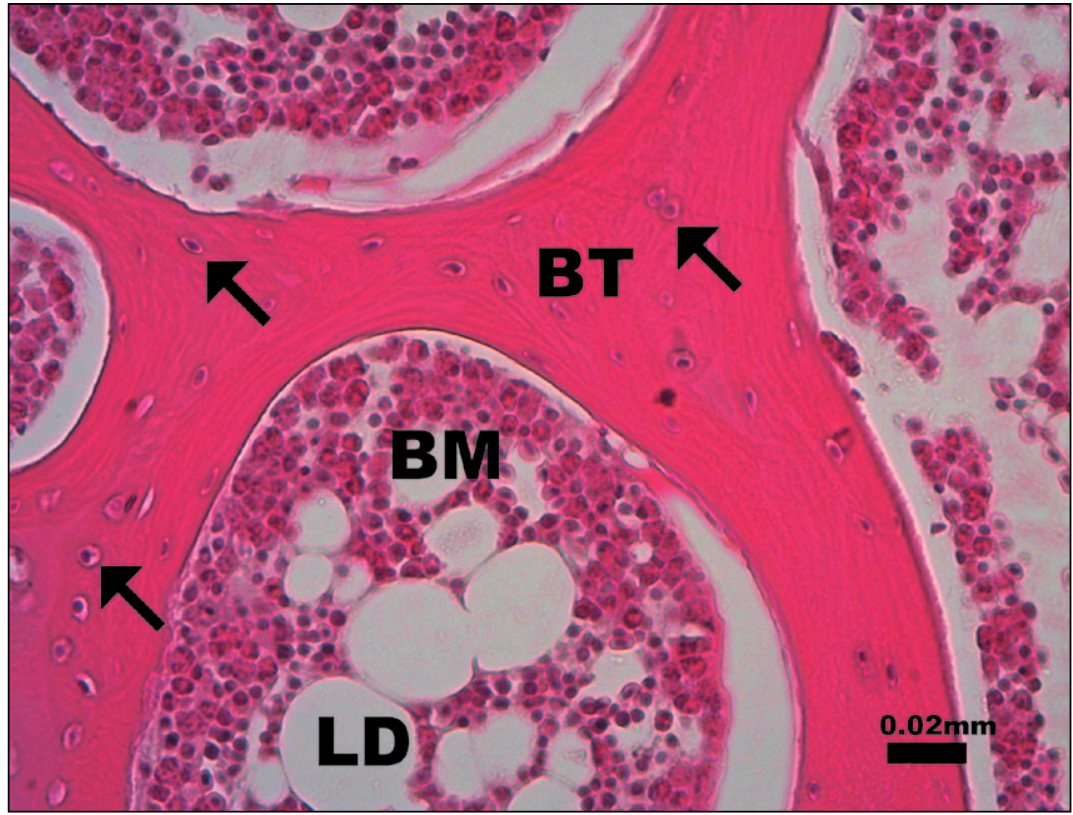

Fig. 3. Section of the spongy bone tissue of control Japanese quail after 63 days of hypodynamy (haematoxylin-eosin staining).

$\mathrm{BT}$ - bone trabeculae, BM - bone marrow, LD - lipid droplets, arrow - osteocyte

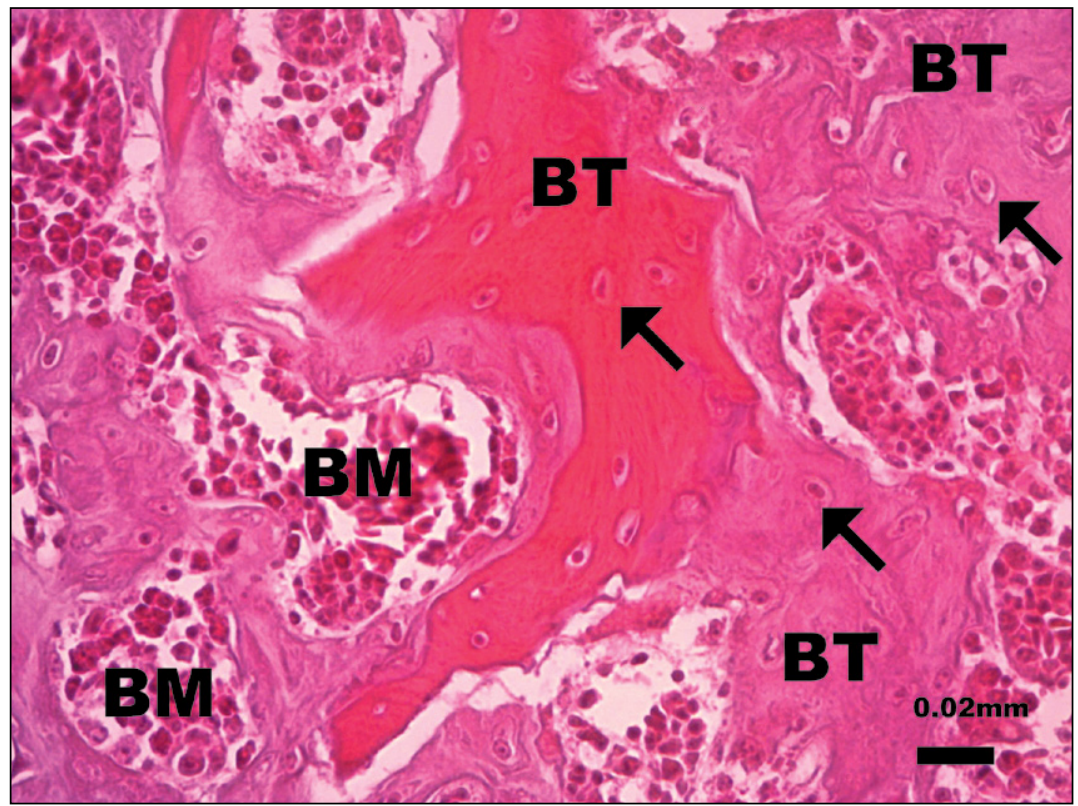

Fig. 4. Section of the spongy bone tissue of experimental Japanese quail after 63 days of hypodynamy (haematoxylin-eosin staining).

$\mathrm{BT}$ - bone trabeculae, BM - bone marrow, arrow - osteocytes 


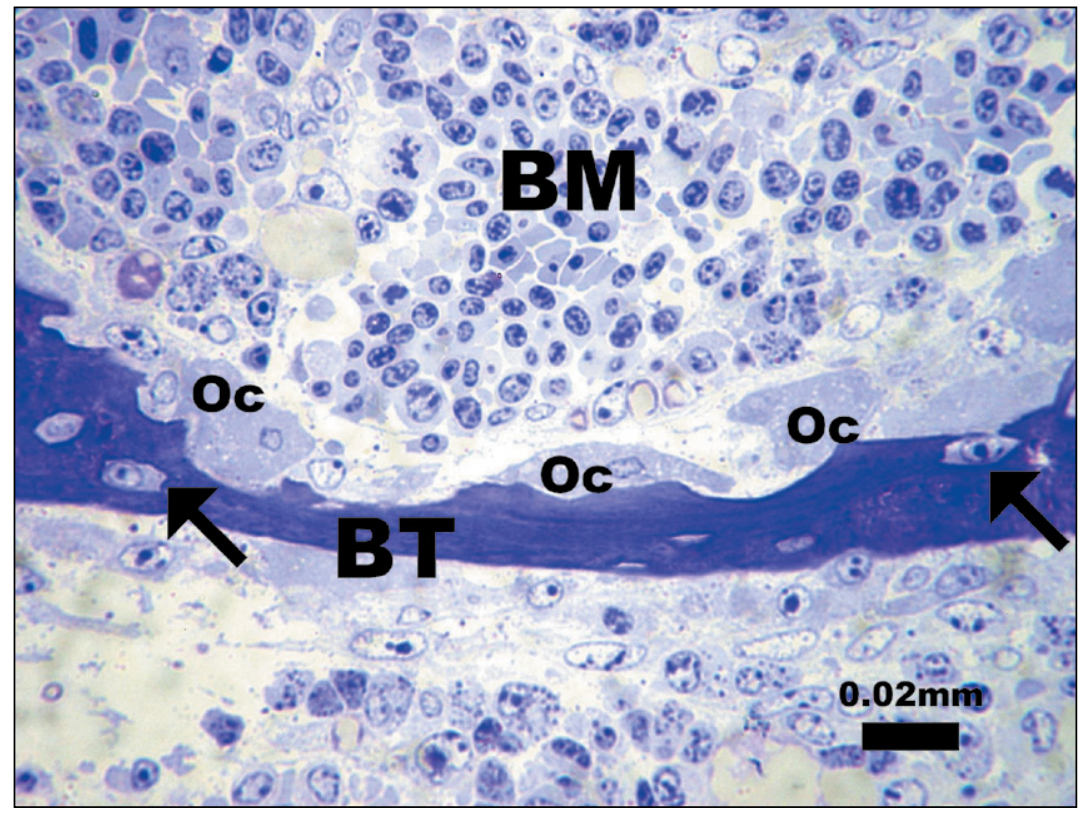

Fig. 5. Semi-thin section of the spongy bone tissue of experimental Japanese quail after 90 days of hypodynamy (toluidine-blue staining).

$\mathrm{BT}$ - bone trabeculae, Oc - osteoclasts, BM - bone marrow, arrow - osteocytes

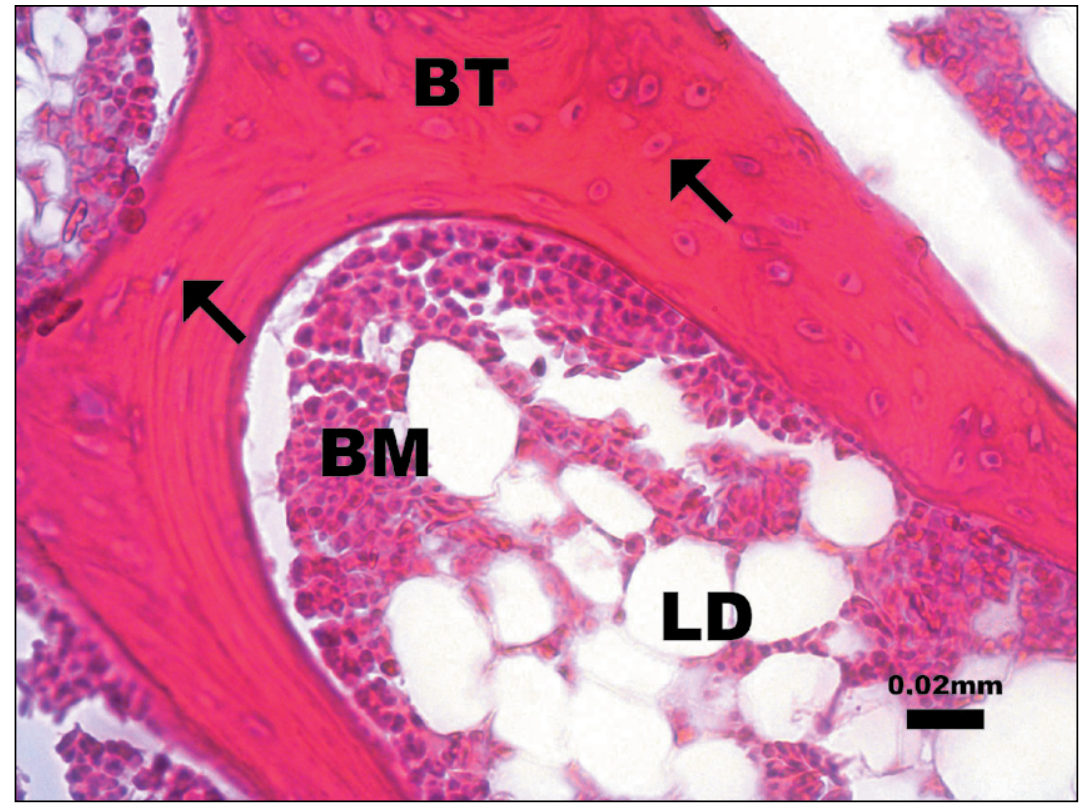

Fig. 6. Section of the spongy bone tissue of control Japanese quail after 180 days of hypodynamy (haematoxylin-eosin staining).

$\mathrm{BT}$ - bone trabeculae, BM - bone marrow, LD - lipid droplets, arrow - osteocytes 


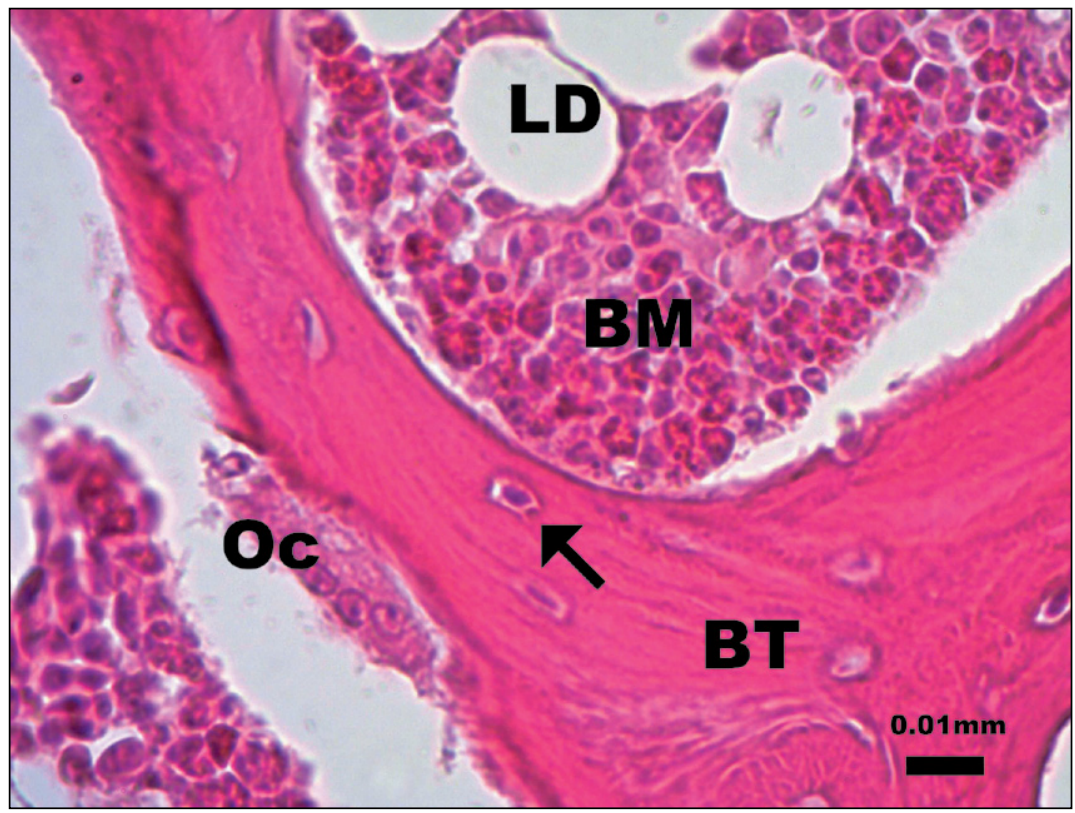

Fig.7. Section of the spongy bone tissue of experimental Japanese quail after 180 days of hypodynamy (haematoxylin-eosin staining).

$\mathrm{BT}$ - bone trabeculae, Oc - osteoclast, BM - bone marrow, LD - lipid droplets, arrow - osteocyte

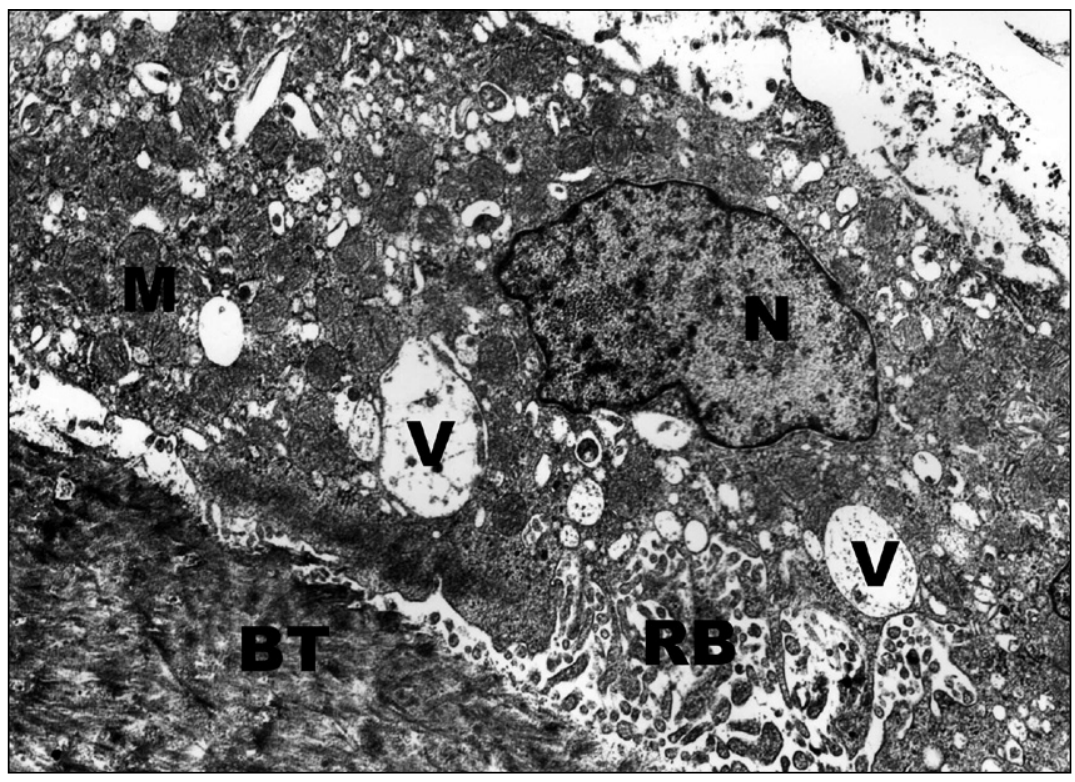

Fig. 8. Electron micrograph of osteoclast of the spongy bone tissue of experimental Japanese quail after 90 days of hypodynamy; ultrathin section ( $\times 6200$ magnification).

$\mathrm{RB}$ - ruffled border; $\mathrm{BT}$ - bone trabeculae, $\mathrm{V}$ - vacuoles and vesicles, $\mathrm{M}$ - mitochondria, $\mathrm{N}$ nucleus 